\title{
Response of $\mathrm{LaBr}_{3}(\mathrm{Ce})$ scintillators to $2.5 \mathrm{MeV}$ fusion neutrons
}

C. Cazzaniga ${ }^{1,2}$, M. Nocente ${ }^{1,2}$, M. Tardocchi ${ }^{2}$, G. Croci ${ }^{2}$, L. Giacomelli $^{1}$, M. Angelone ${ }^{3}$, M. Pillon ${ }^{3}$, S. Villari ${ }^{3}$, A.Weller ${ }^{4}$, L. Petrizzi ${ }^{5}$, G. Gorini ${ }^{1,2}$, the ASDEX UpGrade team and JET-EFDA Contributors*

JET-EFDA, Culham Science Centre, Abingdon OX14 3DB, United Kingdom

${ }^{1}$ University of Milano Bicocca, Piazza della Scienza 3, Milano 20125, Italy

${ }^{2}$ Istituto di Fisica del Plasma, Associazione EURATOM-ENEA-CNR, via Roberto Cozzi 53, Milano 20125 , Italy

${ }^{3}$ Associazione EURATOM-ENEA sulla Fusione ENEA C.R. Frascati, Via E. Fermi, 45, 00044 Frascati (Roma), Italy

${ }^{4}$ Max-Planck-Institut fuer Plasmaphysik, IPP-Euratom Association, Boltzmann str. 2, D-85748 Garching, Germany

${ }^{5}$ IAEA Representative at OECD Nuclear Energy Agency 12, Boulevard des Îles, F-92130 Issy-lesMoulineaux, France

* See the Appendix of F. Romanelli et al., Proceedings of the 24th IAEA Fusion Energy Conference 2012, San Diego, USA

\begin{abstract}
Measurements of the response function of $\mathrm{LaBr}_{3}(\mathrm{Ce})$ to $2.5 \mathrm{MeV}$ neutrons have been carried out at the Frascati Neutron Generator and at tokamak facilities with deuterium plasmas. The observed spectrum has been interpreted by means of a MCNP model. It is found that the main contributor to the measured response is neutron inelastic scattering on ${ }^{79} \mathrm{Br},{ }^{81} \mathrm{Br}$ and ${ }^{139} \mathrm{La}$. An extrapolation of the count rate response to $14 \mathrm{MeV}$ neutrons from deuterium-tritium plasmas is also presented. The results are of relevance for the design of $\gamma$-ray diagnostics of fusion burning plasmas.
\end{abstract}

carlo.cazzaniga@mib.infn.it 


\section{Introduction}

One of the primary goals of next step magnetic fusion devices is the understanding and control of a burning plasma. There is wide consensus among plasma physicists that the dynamics of a plasma close to ignition is largely dominated by the behavior of suprathermal particles [1], which can be generated as products of the main fusion reactions (such as $\alpha$ particles from the $d+t \rightarrow n+\alpha$ reaction) or by external auxiliary heating. For this reason, the distribution function of the energetic ions, that are confined by the magnetic field in the plasma, needs to be measured. $\gamma$-ray spectroscopy is one of the few diagnostic techniques proposed for this scope [2]. Gamma-ray emission results from interactions between the energetic ions and impurities that are naturally found in the plasma [2-4]. Recently, $\gamma$-ray measurements at low counting rates and high energy resolution in present tokamak devices $[5,6]$ have shown that added parameters of the fast ion energy distribution can be obtained by combining information on the intensity and shape of characteristic peaks of $\gamma$-ray reactions occurring in the plasma [7-10]. A review on neutron and gamma-ray measurements in tokamak plasmas for fast ion studies has been recently published in [3].

Unlike present devices, $\gamma$-ray measurements in next step tokamaks, such as ITER, will have to be performed at $\mathrm{MHz}$ counting rates, still with high energy resolution, and under significant irradiation from 2.5 and $14 \mathrm{MeV}$ neutrons produced by the main fusion reactions, $\mathrm{d}+\mathrm{d} \rightarrow \mathrm{n}+{ }^{3} \mathrm{He}$ in deuterium plasmas and $\mathrm{d}+\mathrm{t} \rightarrow \mathrm{n}+\alpha$ in deuterium-tritium. Dedicated solutions therefore need to be developed. A new scintillator material, $\operatorname{LaBr}_{3}(\mathrm{Ce})$ [11-12], meets many of the requirements for $\gamma$-ray measurements at ITER. This detector is resilient to neutron damage and first measurements at low counting rates at tokamak devices have been demonstrated $[10,13]$. High counting rate $\gamma$-ray measurements up to a few $\mathrm{MHz}$ were also shown at nuclear accelerators without any significant degradation of the energy resolution [9]. The effect of 2.5 and $14 \mathrm{MeV}$ neutron irradiation on the detector has not been studied in detail yet, but for one work where the response of a $1.5^{\prime \prime} \mathrm{LaBr}_{3}$ detector to neutrons produced by a conventional ${ }^{241} \mathrm{Am} /{ }^{9} \mathrm{Be}$ source was presented [14]. This result cannot be however easily extrapolated for applications in fusion plasmas, due to the very different neutron spectrum of ${ }^{241} \mathrm{Am} /{ }^{9} \mathrm{Be}$ from that of deuterium and deuterium-tritium reactions.

In this paper we present dedicated measurements of the $\mathrm{LaBr}_{3}$ response to $2.5 \mathrm{MeV}$ neutrons. The experiments were carried out at neutron accelerators and at tokamak devices with deuterium plasmas. The results are analyzed using a MCNP model to identify the main processes contributing to the observed response. Implications of the results for $\gamma$-ray measurements in a deuterium-tritium plasma of ITER are finally discussed.

\section{2. $\mathrm{LaBr}_{3}$ response function to $2.5 \mathrm{MeV}$ mono-energetic neutrons}

Measurements of the $\mathrm{LaBr}_{3}$ response function to $2.45 \mathrm{MeV}$ mono-energetic neutrons were performed at the Frascati Neutron Generator (FNG) [15]. At FNG a deuteron beam was accelerated on a deuterium target, providing a neutron yield of $2 \cdot 10^{8}$ neutrons per second during the measurements. The detector was placed at about $1 \mathrm{~m}$ from the target, which resulted in a neutron fluence on the detector front surface of approximately $8 \cdot 10^{4}$ neutrons per second. The light produced by neutron interactions with the crystal was detected by an eight stage Hamamatsu R6233-01 photo multiplier tube (PMT). The PMT was equipped with a custom developed active base for gain shift minimization at high counting rates [16] and was operated at a high voltage $\mathrm{V}_{\mathrm{HV}}=800 \mathrm{~V}$ (for reference, the PMT nominal gain at $\mathrm{V}_{\mathrm{HV}}=10^{3} \mathrm{~V}$ is $2.7 \cdot 10^{5}$ ). A commercially available 1 Gsample/second, 12 bit digitizer was used to record individual pulses from the PMT. The corresponding pulse height spectrum (PHS) was reconstructed 
off-line using dedicated software [13].

The spectrum measured at FNG is shown in figure 1 and can be compared with that of figure 2 . The latter is the spectrum measured by the same detector in the absence of neutron irradiation and entirely due to its intrinsic radioactivity. $\mathrm{LaBr}_{3}$ has an intrinsic background due to the radioactive ${ }^{138} \mathrm{La}$ isotope (present in trace concentrations of about $0.09 \%$ ) and to actinides. These are manifested as distinctive structures in the spectrum from combined $\alpha, \beta$ and $\gamma$ decays [12]. In the presence of $2.45 \mathrm{MeV}$ neutron irradiation (figure 1), the intrinsic background spectrum cannot be anymore distinguished as neutron interactions with the crystal dominate the response function. The latter is non trivial, as revealed by the rather complex energy spectrum of figure 1, where peaks at several energies appear, the most significant being at $\mathrm{E} \approx 800 \mathrm{keV}$. All structures are found in the region $\mathrm{E}<2.5 \mathrm{MeV}$, whose area is about $90 \%$ of the whole spectrum. In the region $\mathrm{E}>2.5 \mathrm{MeV}$, there is only a roughly exponential tail of events, at a much lower amplitude.

The observed difference between the regions $\mathrm{E}<2.5 \mathrm{MeV}$ and $\mathrm{E}>2.5 \mathrm{MeV}$ can be qualitatively understood on the basis of the processes contributing to the measured response. $2.5 \mathrm{MeV}$ neutrons can interact with the crystal through radiative capture, inelastic and elastic scattering. Of these three mechanisms, only radiative capture and inelastic scattering are of practical importance, as the recoil energy left behind by $2.45 \mathrm{MeV}$ neutron elastic scattering on the heavy ${ }^{139} \mathrm{La},{ }^{79} \mathrm{Br}$ and ${ }^{81} \mathrm{Br}$ isotopes $(\approx$ $100 \mathrm{keV}$ at most) is well below the experimental energy threshold of our measurements $(\approx 500 \mathrm{keV})$. The cross section for neutron inelastic scattering on these three $\mathrm{LaBr}_{3}$ isotopes is presented in figure 3 . Here we do not separate the individual curves corresponding to $\mathrm{LaBr}_{3}$ isotopes left in different excited states after scattering, but we rather show their sum. At $\mathrm{E}_{\mathrm{n}}=2.5 \mathrm{MeV}$, inelastic scattering on ${ }^{79} \mathrm{Br}$, ${ }^{81} \mathrm{Br}$ and ${ }^{139} \mathrm{La}$ is equally likely, with a cross section $\sigma \approx 2 \mathrm{~b}$. For comparison, the cross section for radiative capture is $\sigma \approx 10 \mathrm{mb}$ at $\mathrm{E}_{\mathrm{n}}=2.5 \mathrm{MeV}$, which makes this process of relevance only in the presence of a significant amount of thermalized neutrons. Further processes involving neutron capture with production of charged particles in the exit channel ( $\operatorname{cuch}$ as ${ }^{79} \operatorname{Br}(n, p){ }^{79} \mathrm{Se}$ ) are negligible at all neutron energies, due to their cross sections.

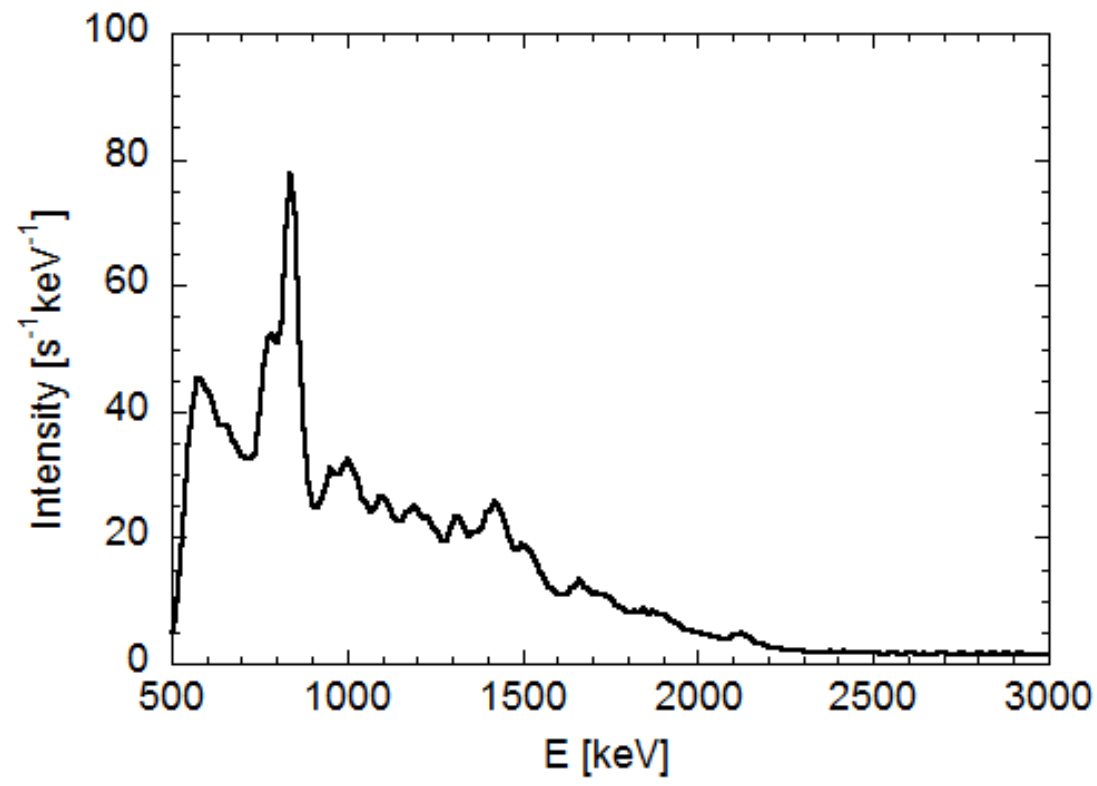

Fig.1: Energy spectrum measured by a 3"x6" $\mathrm{LaBr}_{3}$ detector from mono-energetic $2.5 \mathrm{MeV}$ neutron irradiation at FNG. 


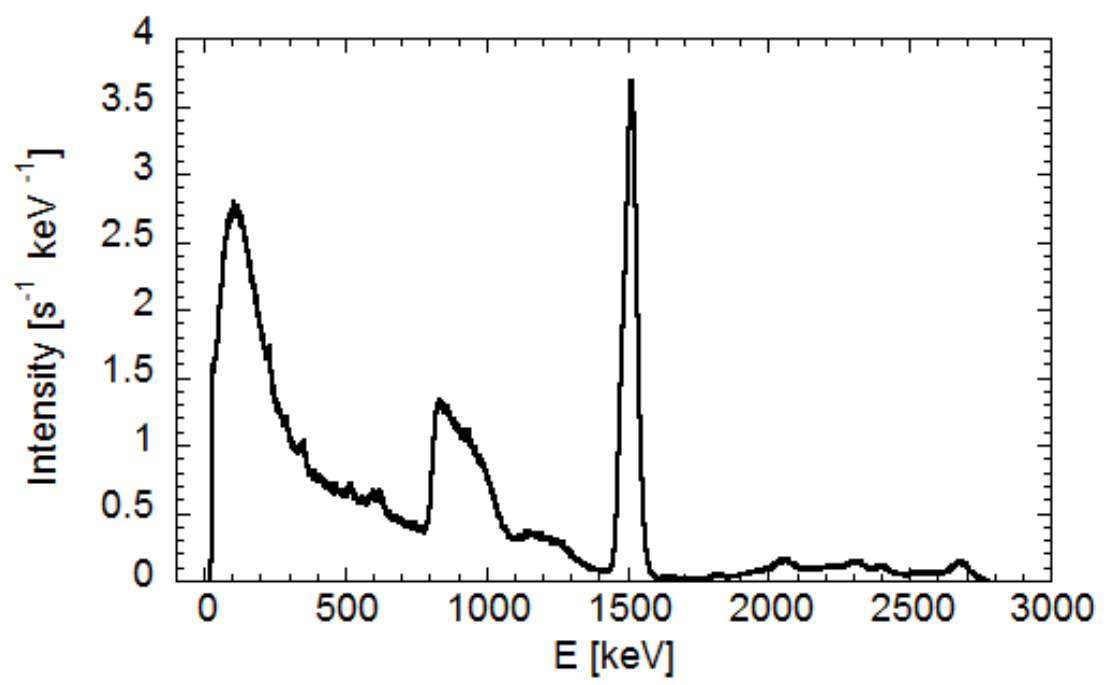

Fig.2: Measured energy spectrum of the intrinsic background of a 3"x6" $\mathrm{LaBr}_{3}$ scintillator.

The spectrum of figure 1 is compared with MCNP simulations in section 4. Here we finally note that neutron inelastic scattering would explain why, as reported by Roberts [14], no neutron/gamma discrimination can be performed with $\mathrm{LaBr}_{3}$. Neutron interactions with this scintillating material also result in $\gamma$-rays. The corresponding pulse shapes could not be thus distinguished from those of external $\gamma$-rays traversing the detector.

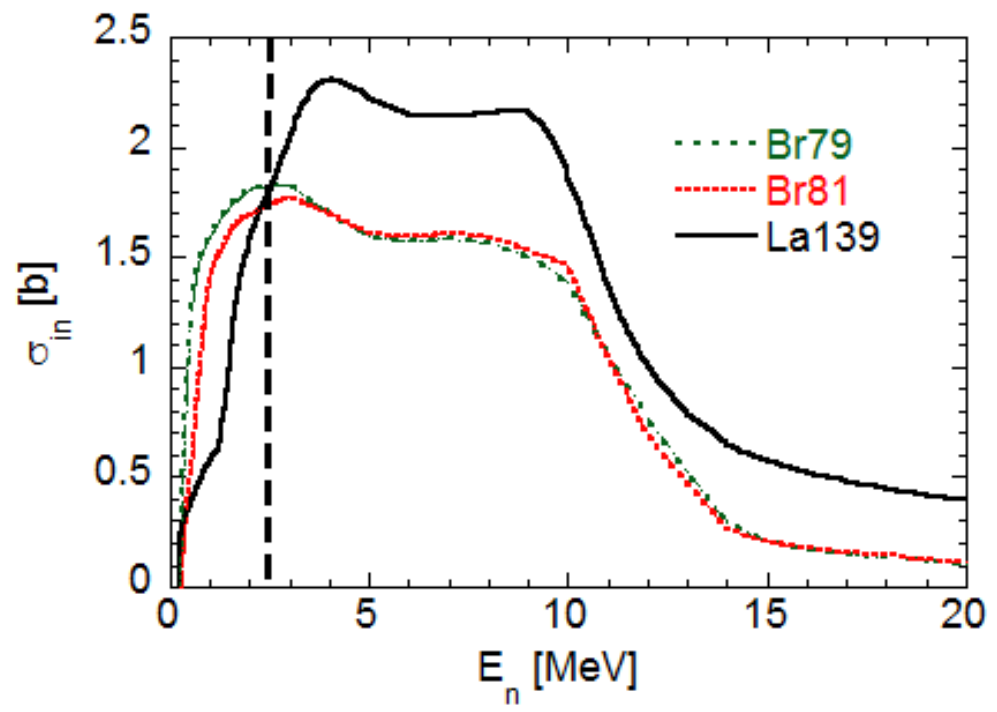

Fig.3 Neutron inelastic scattering cross section on the three $\mathrm{LaBr}_{3}$ isotopes ${ }^{79} \mathrm{Be},{ }^{81} \mathrm{Br}$ and ${ }^{139} \mathrm{La}$ [17]. For each isotope, the summed curve for excitation into any level after neutron scattering is shown. The dashed line corresponds to $2.5 \mathrm{MeV}$. 


\section{2.5 MeV neutron measurements at tokamaks with $\mathrm{LaBr}_{3}$}

The $\mathrm{LaBr}_{3}$ response function to $2.5 \mathrm{MeV}$ from $\mathrm{d}+\mathrm{d}$ reactions was further measured at two different tokamaks, the Joint European Torus (JET) and ASDEX Upgrade (AUG), using the same measurement setup of FNG. Measurements of the scintillator response to fusion neutrons at the AUG tokamak have been first presented in Ref. [18]. In both cases, deuterium plasmas were heated with deuterium neutral beam injection (NBI) at different power levels. In these conditions, the plasma is a good neutron source, where neutron emission mostly arises from reactions between deuterons in the beam and those in the plasma (beam-target reactions). The resulting neutron spectrum is roughly monoenergetic, with an energy spread of about $300 \mathrm{keV}$ around the mean neutron energy $\mathrm{E}_{\mathrm{n}}=2.45 \mathrm{MeV}$ [19]. This makes the experimental conditions of the measurements at JET and AUG comparable to those at FNG. The difference between the two measurements was the position of the detector with respect to the plasma. At AUG, $\mathrm{LaBr}_{3}$ was placed $12 \mathrm{~m}$ from the plasma, along a collimated horizontal line of sight [20], providing a neutron flux at the detector position of $1.7 \cdot 10^{4}$ neutrons $/ \mathrm{sec} / \mathrm{cm}^{2}$. At JET, instead, the distance from the plasma was $23 \mathrm{~m}$, with a collimated vertical view. The neutron flux $\left(0.8 \cdot 10^{4}\right.$ neutrons $/ \mathrm{sec} / \mathrm{cm}^{2}$ ) was here reduced by a factor 2 only with respect to AUG, due to the bigger size of the machine. In both cases, the detector was directly exposed to the $2.5 \mathrm{MeV}$ neutron flux, with no shielding.

In order to verify that the measured signals are dominated by $2.5 \mathrm{MeV} \mathrm{d}+\mathrm{d}$ neutron interactions with $\mathrm{LaBr}_{3}$ (and not, for instance, by nuclear radiation of different origin emitted from the plasma), we can compare the measured counting rate as function of time with variations of the plasma neutron yield, measured by fission chambers (figure 4). The strong correlation between the two traces is a clear indication of the $2.5 \mathrm{MeV}$ neutron origin of the signal. Time variations in the measured traces are due to the specific plasma conditions of the measurements: slower variations can be ascribed to changes in the plasma temperature/density and NBI power; faster variations originate from plasma instabilities, such as sawteeth.

The energy spectra measured at the JET and AUG tokamaks can be compared to the one measured at the FNG neutron source (see Fig. 5). Each spectrum was separately calibrated in energy using radioactive ${ }^{137} \mathrm{Cs}$ and ${ }^{60} \mathrm{Co}$ sources and normalized to an equivalent $150 \mathrm{kHz}$ count rate.

The three spectra show remarkable similarities in terms of peak positions and structures. In each case there are only few events at $\mathrm{E}_{\gamma}>2.5 \mathrm{MeV}$, which confirms the $2.5 \mathrm{MeV}$ neutron origin of the counts recorded in the region $\mathrm{E}_{\gamma}<2.5 \mathrm{MeV}$. The differences between the three spectra can be ascribed to the following effects. The first is the additional contribution of $\gamma$-rays produced by neutrons that interact with materials surrounding the detector. This further background source was different in the three experiments, as it depends on details of the specific environment where the experiment was carried out. A second effect comes from the differences in the neutron energy spectra among the three measurements. At JET and AUG, high energy NBI deuterons reacting with the bulk plasma give rise to neutrons of energy $E_{n}=2.45 \pm 0.3 \mathrm{MeV}$. At the $\mathrm{FNG}$ accelerator, instead, the neutron energy spectrum is narrower around $\mathrm{E}_{\mathrm{n}}=2.45 \mathrm{MeV}$. Moreover, the scattered and moderated components of the neutron spectrum are different depending on details of the line of sight of each experiment. Although these differences, the strong similarity between the measurements strongly indicates that the contribution from $2.5 \mathrm{MeV}$ neutrons interacting with the detector is the dominant one. Finally, we note that neutron induced events mostly lie in the region $\mathrm{E}<2.5 \mathrm{MeV}$, which is promising for $\gamma$-ray observations in deuterium plasmas, as most of the $\gamma$-ray emission from fast ions is expected in the range $2 \mathrm{MeV}<\mathrm{E}_{\gamma}<6 \mathrm{MeV}$. 


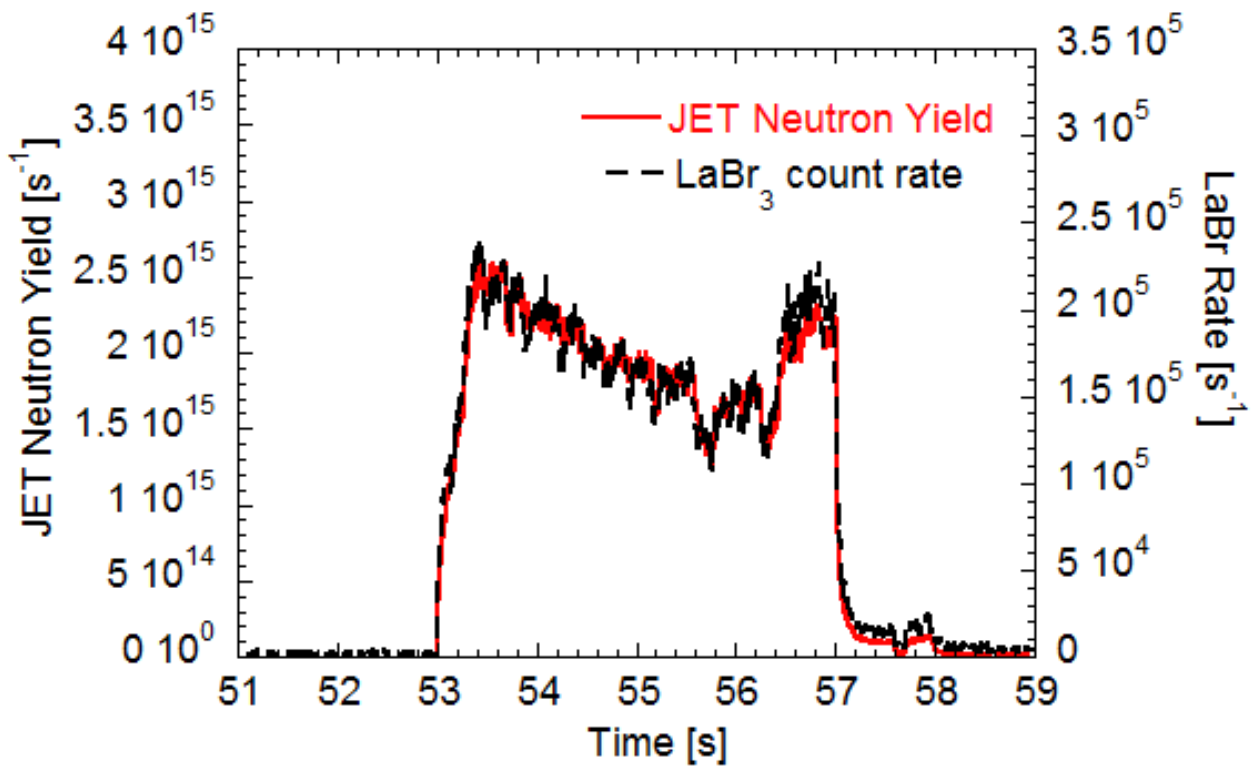

Fig. 4: Time evolution of the counting rate of the $\mathrm{LaBr}_{3}$ spectrometer (dashed line) and JET total neutron yield measured with fission chambers for discharge \#82539 (solid line).

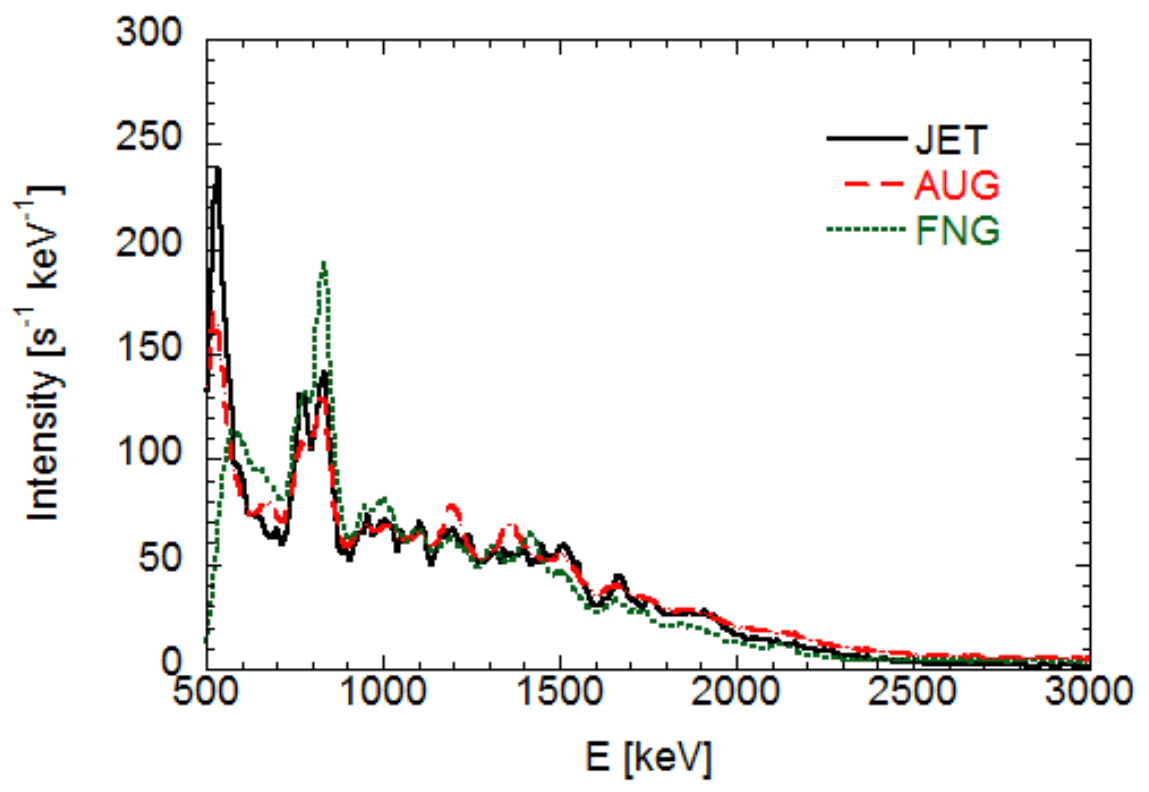

Fig.5: Energy spectra induced by $2.5 \mathrm{MeV}$ fusion neutrons on a $\mathrm{LaBr}_{3}$ scintillator, measured at AUG (dashed), JET (solid) and FNG (dotted). 


\section{Data analysis}

The role of nuclear inelastic scattering from fusion neutrons as the main contributor to the measured response function has been investigated in detail using a MCNP [21] model. The interaction process has been divided into two steps. In the first one, the energy distribution of $\gamma$-rays born from the interaction of a uniform, monoenergetic beam of $2.5 \mathrm{MeV}$ neutrons impinging on the crystal is simulated. In the second step, the resulting neutron induced gamma-ray spectrum is used as input for a new MCNP simulation aimed at evaluating the interaction of these neutron born $\gamma$-rays with the crystal. The continuous-energy neutron data libraries ENDF62MT [22] are used to simulate $\gamma$-rays emitted by neutron interactions with lanthanum and bromine nuclei. The model used in the simulation includes a uniform beam of $2.45 \mathrm{MeV}$ mono-energetic neutrons impinging on the front side of a 3"x6" $\mathrm{LaBr}_{3}$ crystal. No other material is included in the simulation. Fig. 6 separately shows the $\gamma$-ray energy spectrum emitted by each individual isotope ${ }^{139} \mathrm{La},{ }^{79} \mathrm{Br}$ and ${ }^{81} \mathrm{Br}$. The number of excited levels, and therefore the number of corresponding $\gamma$-ray energies from de-excitation, is considerably high. Some of these lines are expected to be overlapped in the measured spectrum, due to the finite energy resolution of the spectrometer, thus appearing as complex structures. A second MCNP simulation was then carried out to take into account the efficiency of the crystal to neutron born $\gamma$-rays of different energy. In this case the output $\gamma$-rays of the first simulation are used and are assumed to be uniformly distributed in the whole crystal volume. Tab. 1 summarize the main results of the simulation. On average, $\mathrm{N}_{\gamma}=1.14 \gamma$-rays per neutron are produced. The fact that $\mathrm{N}_{\gamma}$ is larger than 1 is due to multiple inelastic neutron scattering. In fact, the mean free path of a $2.5 \mathrm{MeV}$ neutron in $\mathrm{LaBr}_{3}$ is about $7 \mathrm{~cm}$, which is about half of the crystal length $(15.24 \mathrm{~cm}=6$ inches $)$. The probability for an emitted $\gamma$-ray to give a signal is 0.7 when the threshold on deposited energy is $\mathrm{E}_{\mathrm{th}}=2 \mathrm{keV}$. This value decreases for higher thresholds; for instance, it becomes 0.65 at $\mathrm{E}>100 \mathrm{keV}$. Therefore we can calculate the detection efficiency to $2.5 \mathrm{MeV}$ neutrons by combining $\mathrm{N}_{\gamma}$ with the $\gamma$-ray detection probability. The result is 0.76 counts per neutron in the region $\mathrm{E}>2 \mathrm{keV}$.

The spectrum obtained with MCNP simulations can be compared to that measured from JET deuterium plasmas (figure 7). An experimental energy broadening has been added to the MCNP simulation for comparison with measurements at JET. Both spectra in figure 7 are normalized to same height. Simulation and measurements are consistent, as the same peaks and structures are found. The small differences observed are due to the following reasons. First of all, we have not included any other material in the simulation but the $\mathrm{LaBr}_{3}$ crystal. Background gamma rays induced by neutron interactions with the tokamak main components and materials surrounding the detector are therefore not accounted for by the MCNP result. For example, the peak at $0.8 \mathrm{MeV}$ can be attributed to the interaction of fast neutrons on iron, an abundant element in most tokamak structures. Similarly, we expect that environmental $\gamma$-rays induced by neutron interactions can fill the gaps in the energy region 1 to $1.5 \mathrm{MeV}$ of the simulated spectrum. The second reason that could explain such differences is that the simulation considers only $2.5 \mathrm{MeV}$ mono-energetic neutrons, and does not include other components of the neutron spectrum. For example, a deuterium plasma can also generate the so-called triton burn up neutrons [23,24]. These are $14 \mathrm{MeV}$ neutrons born from deuterium-tritium reactions (here, tritium is generated in the plasma by $\mathrm{d}+\mathrm{d} \rightarrow \mathrm{p}+\mathrm{t}$ ) and constitute about $1 \%$ of the total neutron emission. The corresponding contribution to the $\mathrm{LaBr}_{3}$ signal is estimated to be about $2 \%$. A more detailed MCNP model could be developed to account for these effects. This goes however beyond the level of accuracy needed for our applications. 


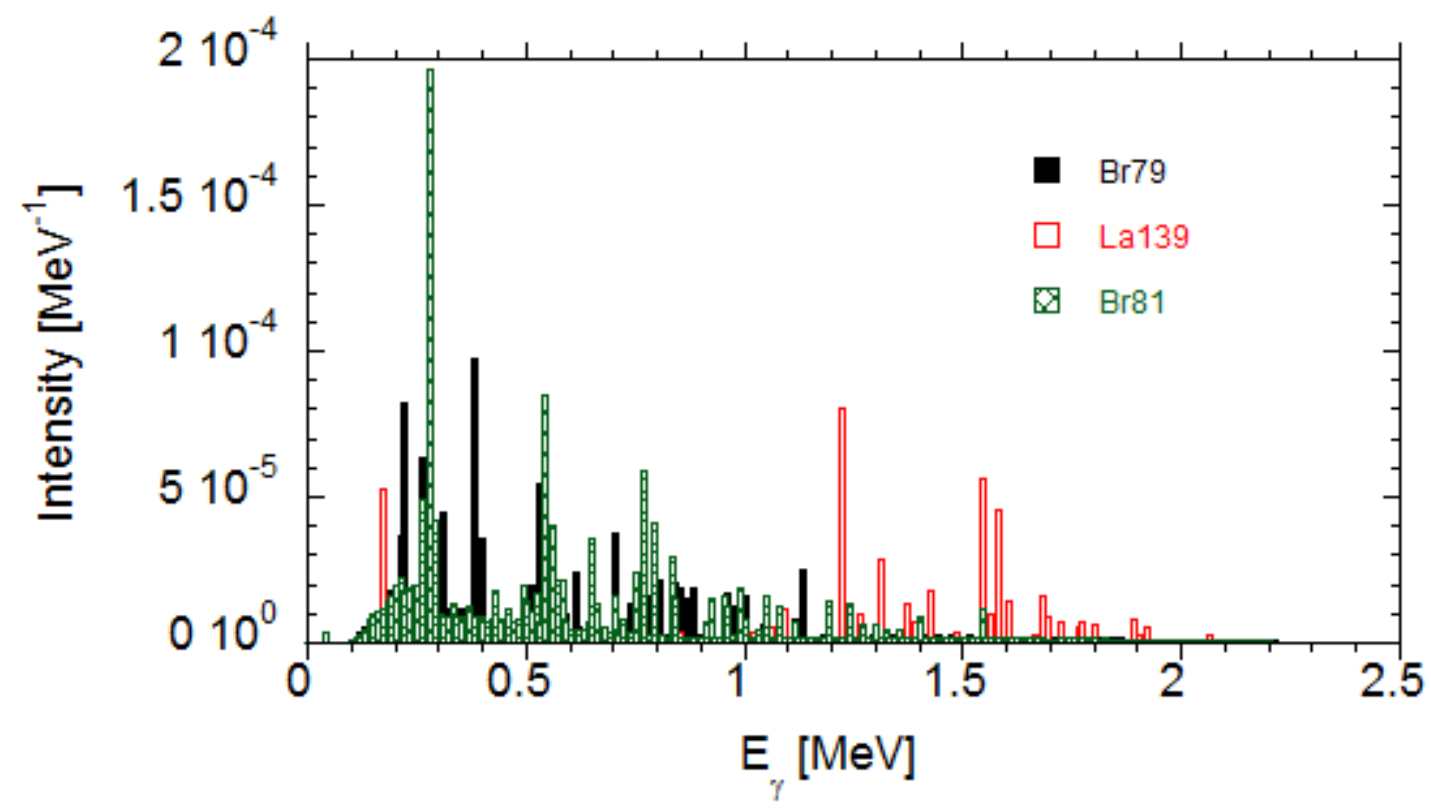

Fig. 6: Energy distribution of $\gamma$-rays induced by $2.5 \mathrm{MeV}$ mono-energetic neutrons simulated with MCNP for a $3 \times 6 \mathrm{LaBr}_{3}$ detector. The contribution of each individual isotope is shown separately.

\begin{tabular}{c|c}
\hline Emitted $\gamma$-rays per neutron & 1.14 \\
Counts per emitted $\gamma$-ray & 0.7 \\
Counts per neutron & $\mathbf{0 . 7 6}$ \\
\hline
\end{tabular}

Tab.1: Summary of the MCNP simulation results for $2.5 \mathrm{MeV}$ neutrons impinging on a 3"x6" $\mathrm{LaBr}_{3}$ detector.

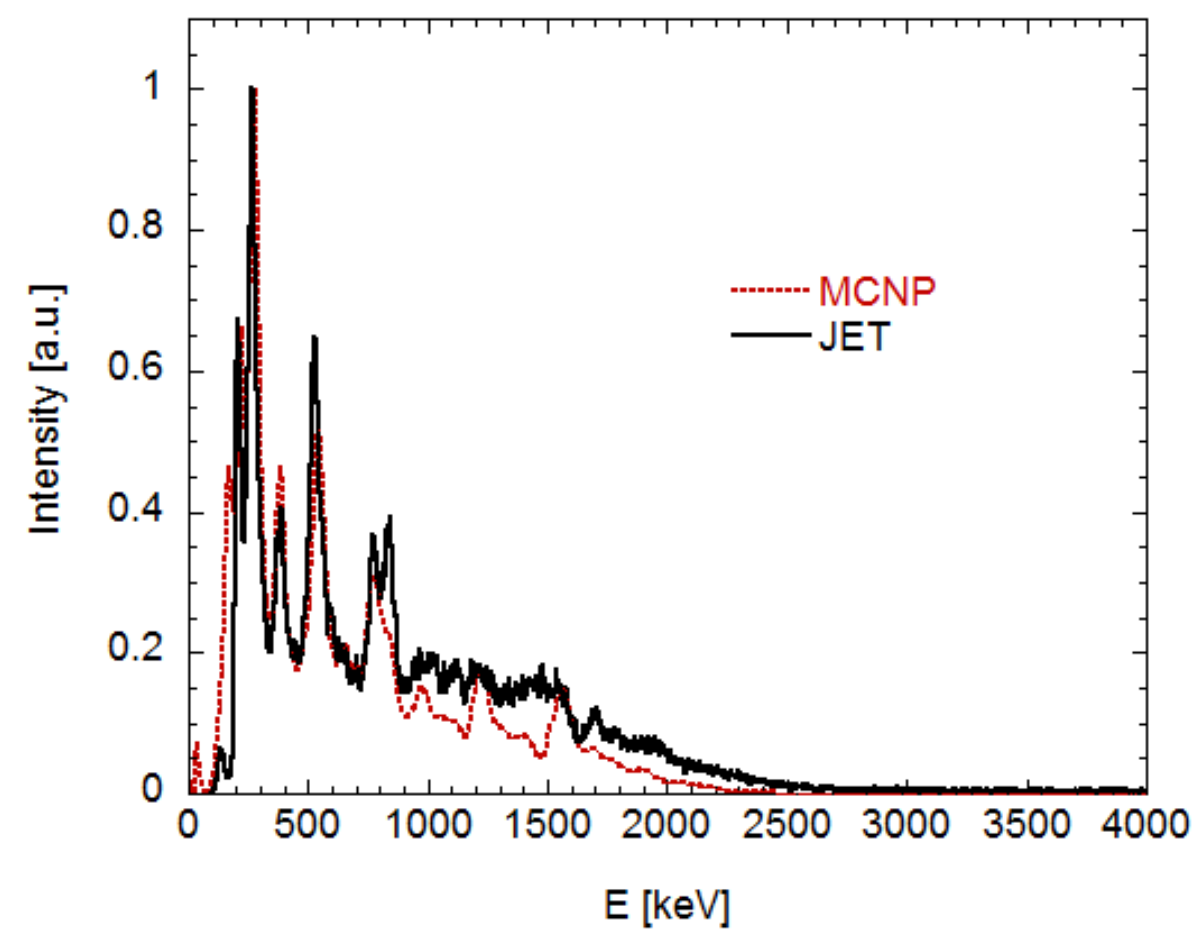

Fig. 7: Response spectrum of a $\mathrm{LaBr}_{3}$ detector to $2.5 \mathrm{MeV}$ neutrons simulated with MCNP and measured at JET. 
As a final test of the MCNP model, we can compare the predicted detector count rate as a function of the total neutron yield of JET with that measured (see Fig.8). Three different discharges have been considered with NBI powers up to $17 \mathrm{MW}$, corresponding to neutron yields as high as $4 \cdot 10^{15} \mathrm{n} / \mathrm{s}$. Measurements have been integrated on time windows of 0.04 seconds. Errors are from counting statistics.

Simulations of neutron transport from the plasma along the detector line of sight [25] predict the ratio of neutron fluence on the detector to the total neutron yield of JET to be $(1.36 \pm 0.4) \cdot 10^{-10}$. This value is known with $30 \%$ uncertainty as it can vary depending on plasma shapes and neutron emission profiles. According to the MCNP simulation, the number of counts on the detector per incoming neutron is 0.76 , of which $88 \%$ are above the $200 \mathrm{keV}$ threshold used in the measurement. For this reason the predicted linear function relating the $\mathrm{LaBr}_{3}$ count rate to the total JET neutron yield has a slope of $0.76 \cdot 0.88 \cdot(1.36 \pm 0.4) \cdot 10^{-10}=(0.9 \pm 0.3) \cdot 10^{-10}$, while the predicted intercept is the intrinsic background count rate $1.5 \cdot 10^{3}$ of the $\mathrm{LaBr}_{3}$ crystal.

\begin{tabular}{c|cc} 
& Measurements (linear fit) & Predicted from simulations \\
\hline Slope & $0.88 \cdot 10^{-10}$ & $(0.9 \pm 0.3) \cdot 10^{-10}$ \\
Intercept & $2 \cdot 10^{3}$ & $1.5 \cdot 10^{3}$
\end{tabular}

Tab. 2: Parameters of the linear function relating the $\mathrm{LaBr}_{3}$ count rate to the total JET neutron yield, as obtained from measurements (left) and simulations (right).

Tab.2 compares the parameters of the linear function predicted by the simulations to the same parameters obtained from a linear fit of the experimental data in figure 8. Results are in good agreement. The scattering of the data points around the best fit line is mostly due to neutron transport to the detector, that depends on plasma shapes and neutron emission profiles (See details in [25]). The dashed lines in the figure correspond to the $\pm 30 \%$ uncertainty on the predicted slope of the $\operatorname{LaBr}_{3}$ count rate as a function of the total JET neutron yield. The scattering of the experimental data lies within these limits.

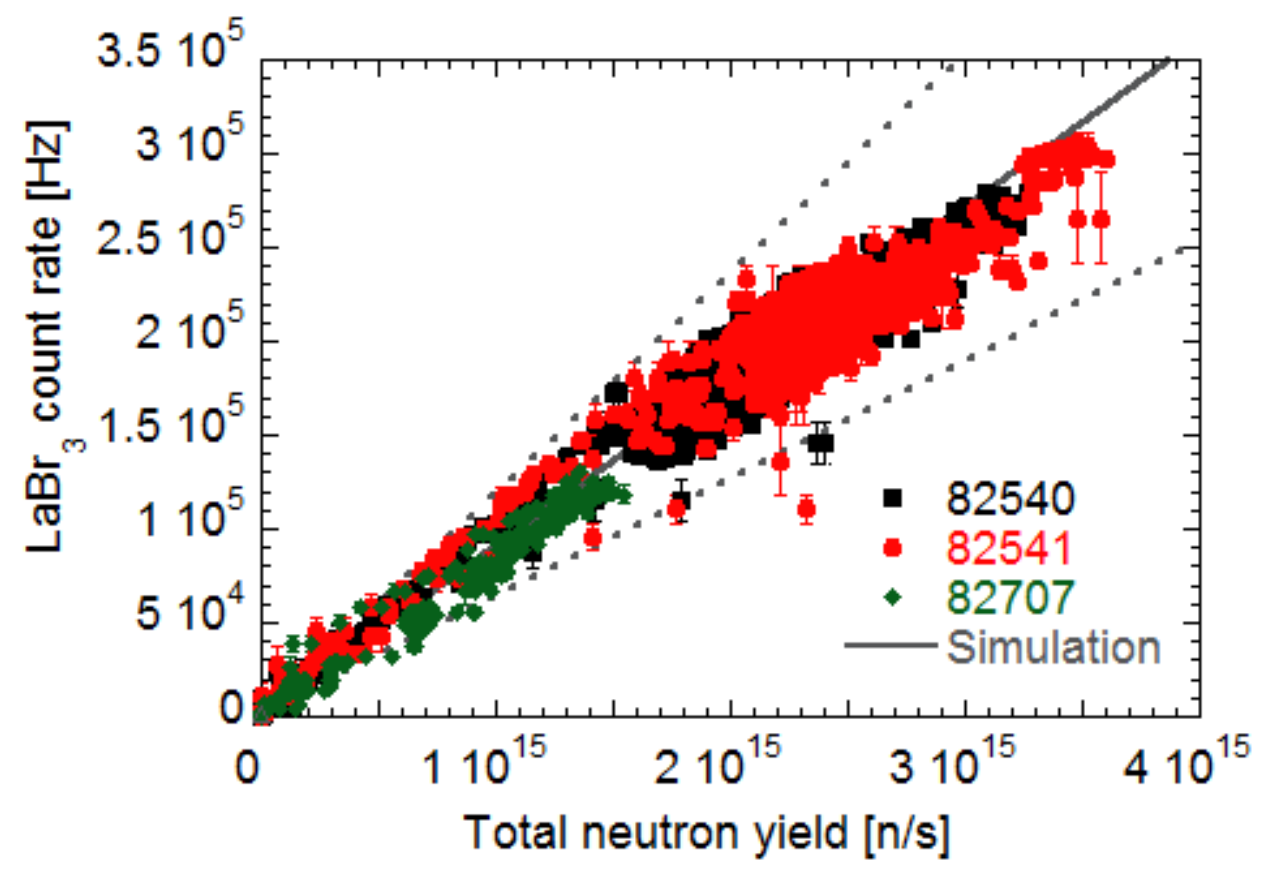

Fig. 8: $\mathrm{LaBr}_{3}$ detector count rate as a function of the JET total neutron yield. Scattered points are from different JET discharges. The dashed lines correspond to the uncertainty on the slope for the linear 
relation between the $\mathrm{LaBr}_{3}$ counting rate and the JET neutron yield, as predicted by simulations.

\section{LaBr response to $14 \mathrm{MeV}$ neutrons from DT reactions}

Based on the results for $2.5 \mathrm{MeV}$ neutrons, we can discuss the expected $\mathrm{LaBr}_{3}$ response function to 14 $\mathrm{MeV}$ neutrons from deuterium-tritium plasmas. More nuclear processes play a role at this neutron energy. Although inelastic scattering cross sections are reduced for $14 \mathrm{MeV}$ neutrons with respect to $2.45 \mathrm{MeV}$ neutrons by a factor 3 (see Fig. 3), Fig. 9 shows that (n,2n) reactions can occur at $14 \mathrm{MeV}$. These are threshold reactions that are active above $9 \mathrm{MeV}, 10 \mathrm{MeV}$ or $11 \mathrm{MeV}$ for ${ }^{139} \mathrm{La},{ }^{81} \mathrm{Br}$ and ${ }^{79} \mathrm{Br}$, respectively, with an increasing cross section as a function of the mass number $\mathrm{A}$. The two neutrons resulting from each $(n, 2 n)$ reaction can be themselves a source of signal, since they have enough energy to undergo inelastic scattering reactions with the other nuclei into the large crystal volume. The nuclei left behind by $(n, 2 n)$ process, which are produced at mass number A-1, are generated in an excited state and, in turn, de-excite by emission of $\gamma$-rays. For this reason, a higher counting rate can be expected from $14 \mathrm{MeV}$ neutrons than from $2.5 \mathrm{MeV}$. Besides, the A-1 nuclei ${ }^{78} \mathrm{Br}$ and ${ }^{80} \mathrm{Br}$ bred by (n,2n) reactions are unstable and undergo a $\beta$ decay with a half life of 6 and 17 minutes, respectively. Their activity can be ignored for short discharges (say, a few seconds), such as those at JET, but will give a large contribution to the background counting rate for long tokamak discharges, like those expected at ITER.

Preliminary MCNP simulations have been performed for $14 \mathrm{MeV}$ neutrons using the same method and simplifications discussed for $2.5 \mathrm{MeV}$. In the model, $14 \mathrm{MeV}$ neutrons are impinging on the front side of a 3"x6" $\mathrm{LaBr}_{3}$ crystal. A first simulation is carried out to determine the number of photons emitted per impinging neutron via inelastic scattering or following a $(n, 2 n)$ reaction. The number of neutrons that undergo $(n, 2 n)$ reactions also gives the number of radioactive nuclei produced. The results of these simulations are presented in Tab. 3. Using these values we can make a first prediction of the detector counting rate. Here we note that, when the neutron flux is not constant as a function of time, the corresponding $\mathrm{LaBr}_{3}$ counting rate is not necessarily proportional to the instantaneous flux. The reason is that the amount of radiation from $\beta$ decays of ${ }^{78} \mathrm{Br}$ and ${ }^{80} \mathrm{Br}$ at time $\mathrm{t}$ depends on the number of radioactive A-1 nuclei produced at time $t-\Delta t$ and is thus proportional to the neutron flux at that time. For the sake of clarity, we shall here assume a constant neutron flux and we limit the estimation of the counting rate to the cases of a very short pulse $(\mathrm{t}<<1 \mathrm{~min})$ and a very long one $(\mathrm{t}>>20 \mathrm{~min})$. The number of counts we obtain per $14 \mathrm{MeV}$ neutron is 1.3 and 1.7 in the two cases, respectively. An energy threshold of $\mathrm{E}_{\gamma}=2 \mathrm{keV}$ in the $\gamma$-ray spectrum is assumed.

Preliminary experiments at FNG were also dedicated to measurements of background induced by 14 $\mathrm{MeV}$ neutrons. The fluence to the detector was $3.8 \cdot 10^{5}$ neutrons $\mathrm{s}^{-1}$ and the measured detector count rate was $5.2 \cdot 10^{5} \mathrm{~s}^{-1}$, in the limit case of a short pulse. This yields 1.4 counts per $14 \mathrm{MeV}$ neutron, which is in agreement with the predicted value (1.3) within $10 \%$.

The measured energy spectrum of a 3"x6" $\mathrm{LaBr}_{3}$ detector is shown in Fig. 10. The calibration was obtained from ${ }^{60} \mathrm{Co}$ and ${ }^{137} \mathrm{Cs}$ laboratory sources. Several structures are observed, which are the result of $\gamma$-rays induced by neutron interactions. The spectrum is however rather flat at high energies $\left(\mathrm{E}_{\gamma}>3\right.$ $\mathrm{MeV}$ ). This is again promising in view of $\gamma$-ray measurements for fusion plasma diagnostics, as $\gamma$-rays from plasma reactions are mostly expected in the range $2 \mathrm{MeV}<\mathrm{E}_{\gamma}<6 \mathrm{MeV}$. 


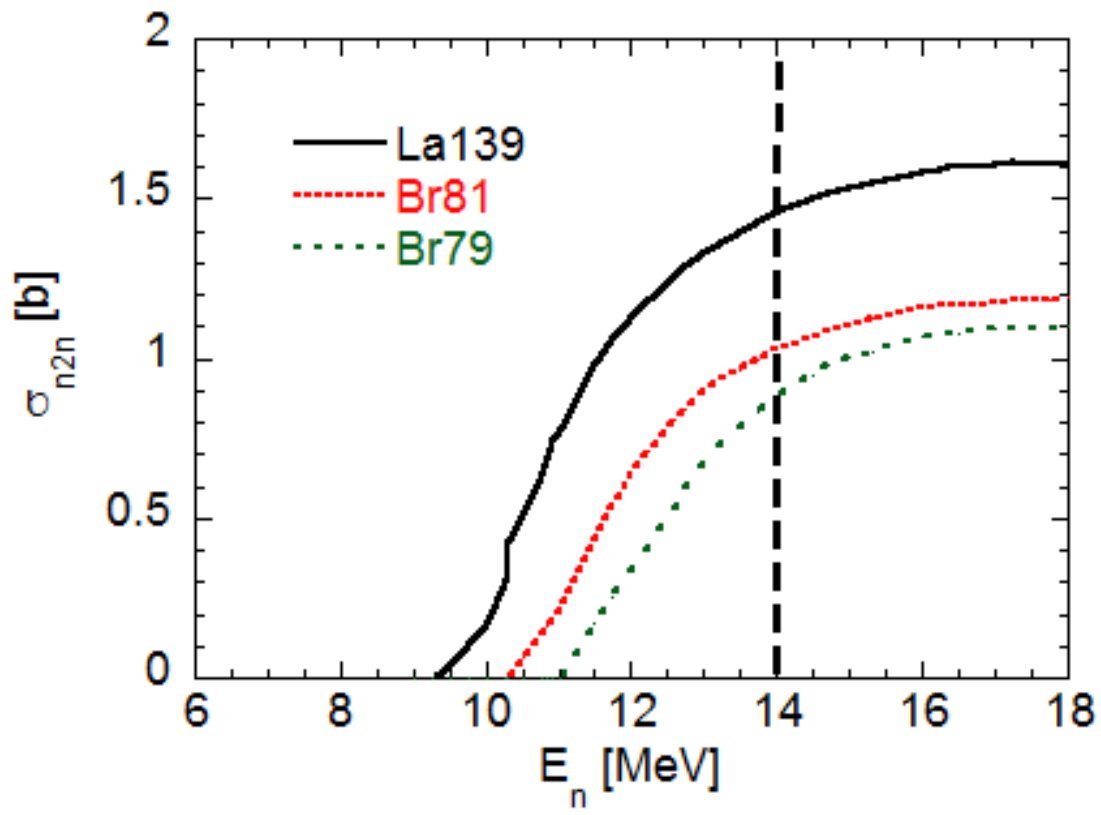

Fig. 9: $(n, 2 n)$ reaction cross sections for the three isotopes of $\mathrm{LaBr}_{3}$ [17]. The dashed line corresponds to $14 \mathrm{MeV}$.

\begin{tabular}{l|l}
\hline$(\mathrm{n}, 2 \mathrm{n})$ reactions per neutron & 0.70 \\
Emitted $\gamma$-rays per neutron & 1.87 \\
Radioactive ${ }^{78} \mathrm{Br}$ nuclei produced per neutron & 0.23 \\
Radioactive ${ }^{80} \mathrm{Br}$ nuclei produced per neutron & 0.23 \\
Counts per neutron $(\mathrm{t}<<\mathbf{1}$ min) & $\mathbf{1 . 3}$ \\
Counts per neutron $(\mathrm{t}>\mathbf{2 0} \mathbf{m i n})$ & $\mathbf{1 . 7}$ \\
\hline
\end{tabular}

Tab. 3: Summary of MCNP simulation results considering $14 \mathrm{MeV}$ neutrons on a 3"x6" $\mathrm{LaBr}_{3}$ detector. 


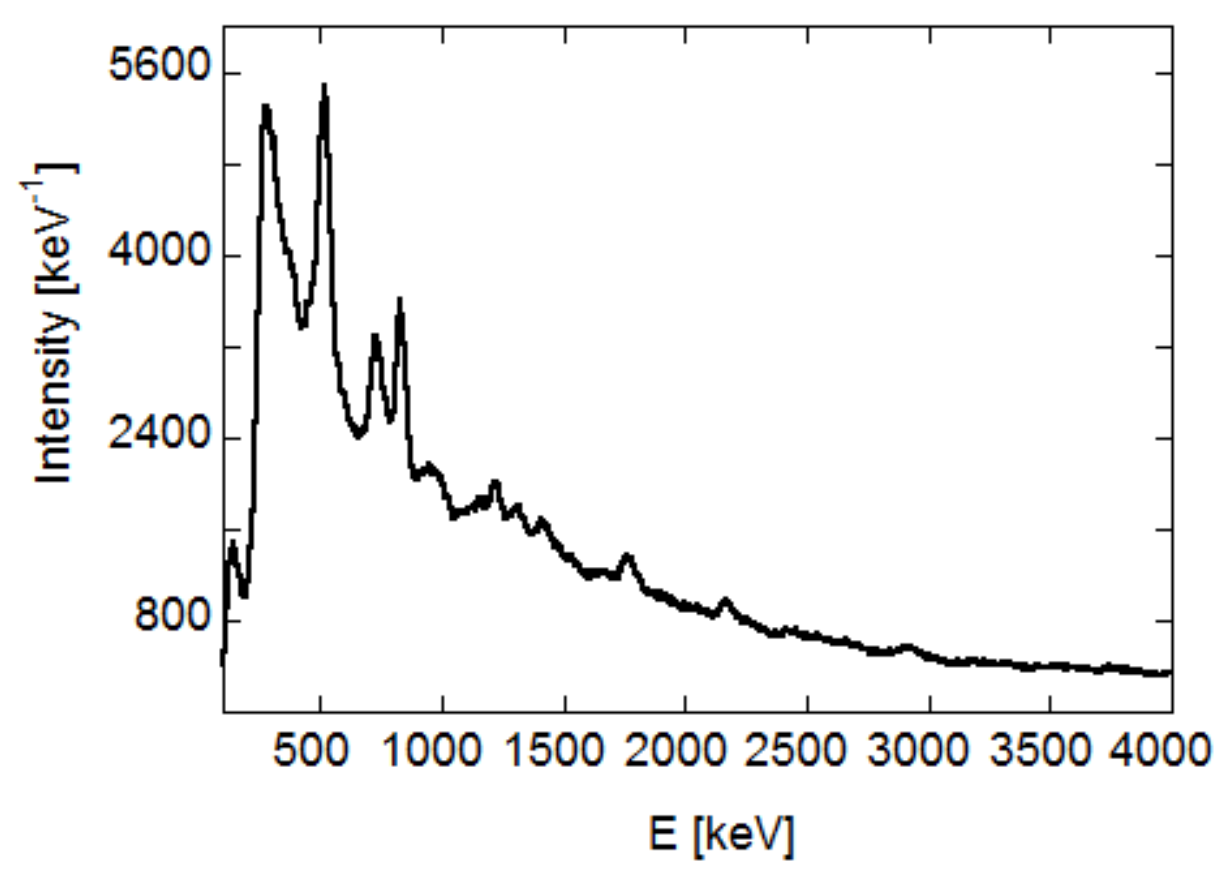

Fig. 10: Energy spectrum measured at FNG using $14 \mathrm{MeV}$ fusion neutrons on a $\mathrm{LaBr}_{3}$ scintillator.

\section{Outlook and implications for ITER}

At ITER, $\gamma$-ray spectroscopy has been proposed for fast ion diagnostics in high power plasmas and, in particular, for $\alpha$ particle diagnosis. $\mathrm{LaBr}_{3}$ is currently the most promising scintillator given its high rate capability and resistance to neutron damage. The possibility to implement a $\gamma$-ray camera system composed of an array of $\mathrm{LaBr}_{3}$ crystals is currently under study for ITER [26]. One of the main challenges is the background on the detector produced by high neutron fluxes up to $10^{8}-10^{9}$ neutrons $\mathrm{cm}^{-2} \mathrm{~s}^{-1}$ at the possible detector position without neutron filters. The present work aims at contributing to that particular problem. The fact that, as shown in figure 1, neutron induced background is mostly distributed on the low energy part of the spectrum, is promising for $\gamma$-ray observations from fast ions. However, the overall background count rate of the detector must also be kept sufficiently low, in order not to exceed its few $\mathrm{MHz}$ total count rate capability, leading to paralysis. Further work needs to be addressed to the design of neutron attenuators that can reduce the overall detector load at an acceptable level to enable measurements. A promising candidate is ${ }^{6} \mathrm{LiH}$, as both ${ }^{6} \mathrm{Li}$ and $\mathrm{H}$, being light nuclei, are good neutron moderators. Besides, ${ }^{6} \mathrm{Li}$ is also a neutron absorber, with a capture cross section of $3 \cdot 10^{3} \mathrm{~b}$ for thermalized neutrons. Clearly, a deeper understanding of the $\mathrm{LaBr}_{3}$ response to $14 \mathrm{MeV}$ neutrons is a preliminary requirement for the attenuator design.

\section{Conclusion}

The response function of a 3"x6" $\mathrm{LaBr} 3$ detector to $2.5 \mathrm{MeV}$ neutrons has been measured to understand the relevance of neutron induced background in view of $\gamma$-ray measurements at ITER.

The experiments were carried out at the FNG neutron source and, with deuterium plasmas, at the ASDEX Upgrade and JET tokamaks, showing a remarkable similarity. A simple MCNP model has been implemented and can reproduce the experimental data both in terms of the main features of the pulse 
height spectrum and expected counting rate. The model reveals that inelastic scattering of $2.5 \mathrm{MeV}$ neutrons with lanthanum and bromine nuclei gives the most important contribution to the response function. Based on the results for $2.5 \mathrm{MeV}$ neutrons, first extrapolations to $14 \mathrm{MeV}$ neutrons show that $(\mathrm{n}, 2 \mathrm{n})$ reactions will play a significant role at this energy, yielding an increased contribution to the background. The presented results are of relevance for the design of $\gamma$-ray diagnostics of fusion burning plasmas, such as ITER.

\section{Acknowledgements}

This work was supported by EURATOM and carried out within the framework of the European Fusion Development Agreement. The views and opinions expressed herein do not necessarily reflect those of the European Commission.

\section{References}

[1] Fasoli, A., C. Gormenzano, H. L. Berk, B. Breizman, S. Briguglio, D. S. Darrow, N. Gorelenkov et al. "Physics of energetic ions." Nuclear Fusion 47, no. 6 (2007): S264.

[2] Kiptily, V. G., F. E. Cecil, and S. S. Medley. "Gamma ray diagnostics of high temperature magnetically confined fusion plasmas." Plasma physics and controlled fusion 48, no. 8 (2006): R59.

[3] Tardocchi, M., M. Nocente, and G. Gorini. "Diagnosis of physical parameters of fast particles in high power fusion plasmas with high resolution neutron and gamma-ray spectroscopy." Plasma Physics and Controlled Fusion 55.7 (2013): 074014.

[4] Kiptily, V. G., F. E. Cecil, O. N. Jarvis, M. J. Mantsinen, S. E. Sharapov, L. Bertalot, S. Conroy et al. " $\gamma$-ray diagnostics of energetic ions in JET." Nuclear Fusion 42, no. 8 (2002): 999.

[5] Tardocchi, Marco, M. Nocente, I. Proverbio, V. G. Kiptily, P. Blanchard, Sean Conroy, M. Fontanesi et al. "Spectral Broadening of Characteristic $\gamma$-Ray Emission Peaks from $\left.{ }^{\wedge}\{12\} \mathrm{C}^{\wedge}\{3\} \mathrm{He}, \mathrm{p} \gamma\right)^{\wedge}\{14\} \mathrm{N}$ Reactions in Fusion Plasmas." Physical review letters 107, no. 20 (2011): 205002.

[6] Nocente, Massimo, Marco Tardocchi, V. G. Kiptily, Patrick Blanchard, I. Chugunov, Sean Conroy, T. Edlington et al. "High-resolution gamma ray spectroscopy measurements of the fast ion energy distribution in JET 4He plasmas." Nuclear Fusion 52, no. 6 (2012): 063009.

[7] Cecil, F. E., and David E. Newman. "Diagnostics of high temperature deuterium and tritium plasmas by spectrometry of radiative capture reactions." Nuclear Instruments and Methods in Physics Research 221, no. 2 (1984): 449-452.

[8] Proverbio, I., M. Nocente, V. G. Kiptily, M. Tardocchi, G. Gorini, and JET-EFDA Contributors. "The $12 \mathrm{C}(3 \mathrm{He}, \mathrm{p} \gamma) 14 \mathrm{~N}$ reaction cross section for $\gamma$-ray spectroscopy simulation of fusion plasmas." Review of scientific instruments 81, no. 10 (2010): 10D320-10D320.

[9] Nocente, M., M. Tardocchi, A. Olariu, S. Olariu, R. C. Pereira, I. N. Chugunov, A. Fernandes et al. "High Resolution Gamma Ray Spectroscopy at MHz Counting Rates With LaBr Scintillators for 
Fusion Plasma Applications." Nuclear Science, IEEE Transactions on 60, no. 2 (2013): 1408-1415.

[10] Nocente, M., M. Garcia-Munoz, G. Gorini, M. Tardocchi, A. Weller, S. Akaslompolo, R. Bilato et al. "Gamma-ray spectroscopy measurements of confined fast ions on ASDEX Upgrade." Nuclear Fusion 52, no. 9 (2012): 094021.

[11] Van Loef, E. V. D., P. Dorenbos, C. W. E. Van Eijk, K. W. Krämer, and H. U. Güdel. "Scintillation properties of $\mathrm{LaBr}_{3}: \mathrm{Ce}^{3+}$ crystals: fast, efficient and high-energy-resolution scintillators." Nuclear Instruments and Methods in Physics Research Section A: Accelerators, Spectrometers, Detectors and Associated Equipment 486, no. 1 (2002): 254-258.

[12] Nicolini, R., F. Camera, N. Blasi, S. Brambilla, R. Bassini, C. Boiano, A. Bracco et al. "Investigation of the properties of a $1 " \times 1 " \mathrm{LaBr}_{3}: \mathrm{Ce}$ scintillator." Nuclear Instruments and Methods in Physics Research Section A: Accelerators, Spectrometers, Detectors and Associated Equipment 582, no. 2 (2007): 554-561.

[13] Nocente, M., M. Tardocchi, I. Chugunov, R. C. Pereira, T. Edlington, A. M. Fernandes, D. Gin et al. "Energy resolution of gamma-ray spectroscopy of JET plasmas with a LaBr scintillator detector and digital data acquisition." Review of scientific instruments 81 (2010): 10 D321.

[14] Roberts, Oliver, Pankaj Joshi, David Jenkins, Bob Wadsworth, and Adam Tuff. "Neutron Response of 1.5" LaBr3: Ce Crystal Scintillators for PARIS." (2008).

[15] Martone, M., M. Angelone, and M. Pillon. "The $14 \mathrm{MeV}$ frascati neutron generator." Journal of nuclear materials 212 (1994): 1661-1664.

[16] Tardocchi, M., L. I. Proverbio, G. Gorini, G. Grosso, M. Locatelli, I. N. Chugonov, D. B. Gin et al. "Gamma ray spectroscopy at high energy and high time resolution at JET." Review of Scientific Instruments 79, no. 10 (2008): 10E524-10E524.

[17] Cross Section Database http://atom.kaeri.re.kr/

[18] Cazzaniga, C., G. Croci, L. Giacomelli, G. Grosso, M. Nocente, M. Tardocchi, and G. Gorini. "LaBr 3 scintillator response to admixed neutron and $\gamma$-ray fluxes." Nuclear Instruments and Methods in Physics Research Section A: Accelerators, Spectrometers, Detectors and Associated Equipment (2013).

[19] Hellesen, Carl, M. Albergante, E. Andersson Sundén, Luigi Ballabio, Sean Conroy, Göran Ericsson, M. Gatu Johnson et al. "Neutron spectroscopy measurements and modeling of neutral beam heating fast ion dynamics." Plasma Physics and Controlled Fusion 52, no. 8 (2010): 085013.

[20] Tardini, G., A. Zimbal, B. Esposito, F. Gagnon-Moisan, D. Marocco, R. Neu, and H. Schuhmacher. "First neutron spectrometry measurements in the ASDEX Upgrade tokamak." Journal of Instrumentation 7, no. 03 (2012): C03004.

[21] The MCNPX website: $\underline{\text { http://menpx.lanl.gov/ }}$

[22] Chadwick, M. B., et al. "ENDF/B-VII. 0: Next generation evaluated nuclear data library for nuclear science and technology." Nuclear Data Sheets 107.12 (2006): 2931-3060. 
[23] Ballabio, L., J. Frenje, J. Källne, S. W. Conroy, G. Ericsson, M. Tardocchi, E. Traneus, and G. Gorini. "Measurement and interpretation of the spectrum of the triton burnup neutron emission from deuterium tokamak plasmas." Nuclear fusion 40, no. 1 (2000): 21.

[24] Sjöstrand, Henrik, Giuseppe Gorini, Sean Conroy, Göran Ericsson, Luca Giacomelli, Hans Henriksson, Anders Hjalmarsson et al. "Triton burn-up neutron emission in JET low current plasmas." Journal of Physics D: Applied Physics 41, no. 11 (2008): 115208.

[25] Johnson, M. Gatu, Sean Conroy, Marco Cecconello, E. Andersson Sundén, Göran Ericsson, M. Gherendi, Carl Hellesen et al. "Modelling and TOFOR measurements of scattered neutrons at JET." Plasma Physics and Controlled Fusion 52, no. 8 (2010): 085002.

[26] Chugunov, I. N., A. E. Shevelev, D. B. Gin, V. G. Kiptily, G. Gorini, M. Nocente, M. Tardocchi, D. N. Doinikov, V. O. Naidenov, and E. M. Khilkevitch. "Development of gamma-ray diagnostics for ITER." Nuclear fusion 51, no. 8 (2011): 083010. 